\title{
An evolutionary view of chromatography data systems used in bioanalysis
}

This is a personal view of how chromatographic peak measurement and analyte quantification for bioanalysis have evolved from the manual methods of 1970 to the electronic working possible in 2010. In four decades there have been major changes from a simple chart recorder output (that was interpreted and quantified manually) through simple automation of peak measurement, calculation of standard curves and quality control values and instrument control to the networked chromatography data systems of today that are capable of interfacing with Laboratory Information Management Systems and other IT applications. The incorporation of electronic signatures to meet regulatory requirements offers a great opportunity for business improvement and electronic working.

The evolution of chromatography data systems (CDSs) used in bioanalysis over four decades is reviewed from the personal perspective of the author. The approach will be selective and therefore not all techniques and approaches will be included or discussed. In this paper I will use the term CDS to include (incorrectly) manual methods of peak measurement to ensure complete coverage of techniques used in bioanalysis.

\section{CDS for bioanalysis}

\section{- Where are we now?}

Today, a CDS used in bioanalysis has a number of functions that it can perform and that we take for granted; many are constant over all of the commercial data systems used in our laboratories and others are specific to a particular vendor. Use of a specific function is dependent on the way a system is operated by a particular laboratory and the nature of the chromatographic equipment the CDS controls. Some of the functions used by most CDSs consist of all or most of the following:

- Establishment and storage of analytical methods including data acquisition and processing parameters;

- Instrument control of LC and LC-MS instruments used by the laboratory associated with each analytical method;

- Sequence file to identify individual samples injected and factors used to adjust the calculated concentration for dilution of the sample or salt form of the analyte;
- Acquisition of data from each injection, together with injection number from the autosampler and any chromatographic conditions with storage of the data either on a local workstation or networked server;

- Process the acquired data first into peak areas or heights and then into analyte concentrations;

- Processing of standard curve samples to determine the calibration curve and quantification of the unknown samples;

- Quantification of the quality control samples to determine whether the run is acceptable or not;

- Quantification of the unknown samples from the study to determine the concentration of the parent drug and any metabolites;

- Storage of the resultant processed data files and other information acquired during the run;

- Interface with Laboratory Information Management Systems (LIMS) for import of data relating to CDS set-up or export of data for further processing or collation of results;

- Regulatory compliance features such as security, access control and audit trail;

- Ability to work electronically with the application of electronic signatures at key stages of analysis.

The list above is for a sophisticated electronic application and working environment but as this paper is about the history of CDS used for

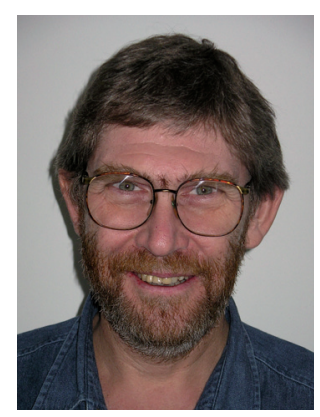

RD McDowall

McDowall Consulting, 73 Murray Avenue, Bromley,

Kent, BRI 3DJ, UK

Tel.: +44 2083130934

E-mail: rdmcdowall@

btconnect.com

\section{FUTURE SCIENCE}


bioanalysis, we have to look back to see how these functions have developed.

\section{- Where have we come from?}

Looking back from today's CDS, we will see an evolutionary journey from manual peak measurement through to an expansion of scope into instrument control, calculation of standard curve parameters, reporting results and interfacing with applications in the laboratory, such as LIMS, which manage the studies, sample management, analytical results and reports.

As background reading for this article the following books are recommended about CDS:

- Norman Dyson: Chromatographic Integration Methods [1]

- RD McDowall: Validation of Chromatographic Data Systems, Meeting Business and Regulatory Requirements [2]

If a comprehensive view of the approaches to and the understanding of chromatographic integration are required the reader should read a further book by Dyson [1].

Although not covered in this paper, the reader should consider in parallel the evolution of bioanalysis in drug discovery and drug development as a background to the evolution of CDS discussion. In the 1970s, the bioanalytical work from preclinical and clinical studies comprised relatively small numbers of samples. Method validation was very simplistic, the techniques for quantification were, in comparison with today, crude and there was little attempt to run quality control samples or check analytical run-to-run variation. In the 1980s, this started to change and larger numbers of samples were generated from studies with guidelines for bioanalytical method validation initiated in the 1990's [3]. Furthermore, good laboratory practice (GLP) regulations have all had an effect on CDS used in bioanalysis. The US GLP regulations were first promulgated in 1978, with little emphasis on computers, but with increased use of computerization in the 1980s, computers were interpreted as equipment and had to be validated and controlled. In 1997, the implementation of Title 21 CFR Part 11 of the Code of Federal Regulations (21 CFR 11) for electronic records and electronic signatures meant that bioanalysis started to change the definition of raw data from paper records to electronic files [4].

\section{The evolutionary ages of CDS}

To provide an overview of the evolution of CDS that I will cover in this paper, I have divided the ages of CDS into four categories: the Stone Age, Bronze Age, Iron Age and Technology Age.

TABLE I describes these four eons that I will discuss in more detail, including the characteristics of each age and the delivery mode. The characteristics present a general description of the CDS functions for each age plus the delivery mode(i.e., the technology used to deliver the CDS at the laboratory bench). Taken overall, it presents the evolution from paper to the current CDS used in our laboratories today. In addition, Figure $\mathbf{I}$ shows the approximate timelines when the different technologies were used; the dates presented are based on personal experience and are not intended to be absolute values.

The major step changes in the evolution are the move from paper to electronic and the introduction of electronic working practices with regulatory compliance that can finally reduce the dependence on paper that has characterized the whole timeline for bioanalysis.

\section{Stone Age: paper-based peak measurement techniques}

At the beginning of the Stone Age, the chromatographic detector signal was recorded on chart paper followed by peak quantification using one of two methods: manual peak measurement by either 'cut and weigh' or 'ruler and pencil'; a third option of counting the squares was also available but not as widely used. In the late Stone Age, the development of the disk integrator automated peak-area measurement but this still required the basic component of Stone Age chromatography: the chart recorder. GC was the predominant technique in the early 1970s as HPLC was still evolving but by the end of the decade, HPLC was the chromatographic technique of choice for bioanalysis.

\section{- Cut \& weigh}

One of the really hands-on jobs for early peak measurement and quantification was cutting and weighing the actual peaks. As the name suggests, this is a relatively simple technique to understand; however, performing it required patience, concentration, a sharp pair of scissors and good hand-eye coordination. After the whole run had been injected, the baselines were drawn underneath each peak and then the whole peak was cut from the chart recorder paper and weighed. This method relies on the accuracy of cutting out the peak, consistent 
Table 1. Four ages of chromatography data systems evolution.

\begin{tabular}{|c|c|c|}
\hline Eon & Characteristics & Delivery mode \\
\hline Stone Age & $\begin{array}{l}\text { Paper-based peak measurement using a chart recorder } \\
\text { Focus only on peak measurement } \\
\text { Manual techniques } \\
\text { Disk integrators provided a degree of automation } \\
\text { No instrument control } \\
\text { No automation of standard curve and unknown } \\
\text { calculations: manual processes }\end{array}$ & $\begin{array}{l}\text { Cut and weigh } \\
\text { Pencil and ruler } \\
\text { Count the squares } \\
\text { Disk integrator }\end{array}$ \\
\hline Bronze Age & $\begin{array}{l}\text { Automation of peak measurement } \\
\text { Some simple calibration models available and analyte } \\
\text { quantification in later models (e.g., response factor and } \\
\text { single point calibration) but these were not very useful } \\
\text { for bioanalysis } \\
\text { Data files saved in operating system directories (mini } \\
\text { computer CDS) } \\
\text { Later integrators could save data files on a network }\end{array}$ & $\begin{array}{l}\text { Integrators (single and } \\
\text { multichannel) } \\
\text { Mini computer-based CDS }\end{array}$ \\
\hline Iron Age & $\begin{array}{l}\text { Automation of peak measurement } \\
\text { Instrument control automated } \\
\text { Dilution and factor calculations } \\
\text { Calculation of calibration curves based on linear } \\
\text { regression models } \\
\text { Custom interface with LIMS } \\
\text { Data files saved in operating system directories } \\
\text { Basic audit trail functions }\end{array}$ & $\begin{array}{l}\text { PC-based CDS } \\
\text { Mini computer CDS }\end{array}$ \\
\hline $\begin{array}{l}\text { Technology } \\
\text { Age }\end{array}$ & $\begin{array}{l}\text { Automation of peak measurement } \\
\text { Instrument control automated } \\
\text { Calculation of standard curve based on linear } \\
\text { regression models } \\
\text { Quality control sample calculations with run } \\
\text { acceptance criteria } \\
\text { Dilution and factor calibrations } \\
\text { Electronic signatures } \\
\text { Configurable interface with LIMS } \\
\text { Data files saved in operating system directories } \\
\text { Database used for data storage of all CDS data } \\
\text { Compliant audit trail functions }\end{array}$ & $\begin{array}{l}\text { PC-based CDS } \\
\text { PC network with central } \\
\text { server for data storage } \\
\text { Terminal server with } \\
\text { central server }\end{array}$ \\
\hline
\end{tabular}

paper density and humidity of the laboratory. However, if a supervisor does not believe that the baseline is correctly placed it is rather difficult to recalculate.

A major problem with cut and weigh is the low dynamic range of any analytical method (and also ruler and pencil); the whole of the peak has to be on the chart recorded. This can be helped by attenuation of the output signal from the chromatograph or scale on the chart recorder, typically with a second and sometimes a third injection; however, it was extremely difficult to obtain linearity across the assay's dynamic range. This technique had been consigned to the dustbin of history but in the 1990s it was revived, rather ironically, for computer system validation as a method useful for testing that the outputs of data systems used for regulated work were correct.

\section{- Ruler \& pencil}

This is the baseline (sorry!) for measuring peaks - it is a slow, manual, labor-intensive and subjective process and placement of the peak baselines is based upon the training and scientific judgement of the chromatographer. Peak height measurement was just the measurement of the height from the drawn baseline to the apex of the peak in millimetres. Peak area was calculated by measuring the peak width at the half height of the peak and multiplying the value with the peak height to obtain the peak area. One advantage of the pencil and ruler approach is that reasoning of baseline placement and peak width measurement are visually recorded on the chromatogram itself, providing an implicit audit trail for others to check both the measurements and the calculations. However, the method requires a sharp pencil and a clear perspex ruler to ensure the best lines are drawn for 


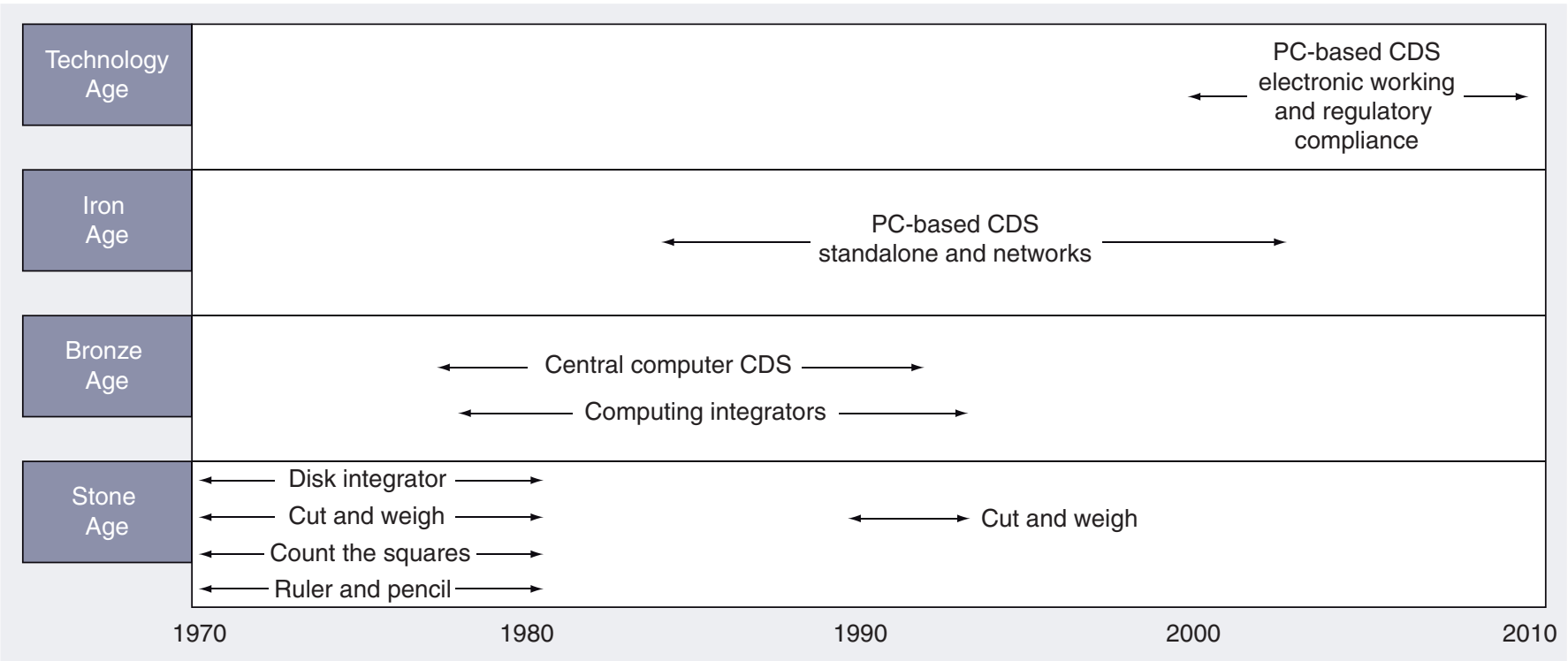

Figure 1. Timeline of the evolution of CDS used in bioanalysis.

CDS: Chromatography data systems.

each peak. More technologically advanced and richer chromatographers could invest in a Vernier calliper to replace the ruler and measure the peak heights and half widths in an atempt to try and measure with more accuracy compared with the ruler. On the other hand you could purchase National Institute of Standards and Technologycalibrated rulers as an alternative approach.

\section{- Count the squares}

If the chromatogram was recorded on squared chart paper, then quantification using a technique called 'count the squares' was used, especially by chromatographers who have had a frontal lobotomy. After drawing the baseline on each peak using a ruler and pencil, the number of squares under each analyte and internal standard peak were counted manually - by all accounts a very labor-intensive and extremely tedious process for both the chromatographer and their supervisor. From the author's perspective, ruler and pencil techniques were far quicker than counting the squares.

\section{- Disk integrator}

One of the first attempts to automate peak measurement resulted in the production of disk integrators linked to chart recorders [1]. Essentially, these were electromechanical devices that linked the movement of the main pen of a chart recorder to a device that converted this movement into a second trace on a calibrated track at the side of the main chart. As the main pen traced the peak rise and fall, the disk integrator moved left and right across the calibrated track; every ten full traces, there was a further movement of the pen (equivalent to 1000 area counts) to help quantify large peaks. Although this removes much of the tedium from peak measurement, a number of problems were found with this approach, such as the need for a stable baseline for accurate measurement (as the quantitative peak trace could otherwise drift over time) and good symmetrical peak shape. However, the great benefit is that peaks did not need to be attenuated to keep them on the chart paper as the disk integrator could handle the peak measurement. Typically, there was a lag between the peak rise on the main chart recorder and the disk integrator response. However, use of a disk integrator speeded up the peak measurement so much so that the peak area counts could be calculated just after an individual injection was completed and there was no need to complete the whole run before peak measurement started.

Chart recorders \& paper chromatograms All Stone Age CDS techniques were dependent on a chart recorder to trace the peak and this could suffer a number of problems, such as:

- Running out of ink or ink flow blocked during an injection;

- Too much ink delivered by the pen, with resulting ink blots in the chromatogram;

- The pen ripping the chart paper. 
However, despite these problems, one key point to make with Stone Age CDS methods is that a chromatographer looked very closely at each chromatogram, the analyte peak shapes and the placement of the baselines. In fact, an experienced chromatographer's eye is often better than any later-age CDS for detecting very subtle changes in peak shape (e.g., co-eluting peaks) that, even today, few CDS algorithms can identify. In addition, it was easier to identify by visual inspection any late eluting peaks that interfered with subsequent injections as there was a continuous plot of the detector output on the chart paper.

\section{- Summary of Stone Age CDS}

The major limitations with all of the Stone Age CDS methods is the sole focus on peak measurement, as there is no encompassing data system coupled with no instrument control and no automated calculation of the standard curve and unknown concentrations. Calculation of all standard curve and peak-area ratios and final analyte concentration were performed manually (plotting a standard curve on a sheet of graph paper) with unknown concentration values read from the resulting best-fit line placed by the bioanalyst's eye. However, looking on the bright side, there was no need to bother with computer validation.

\section{Bronze Age: electronic peak measurement}

The middle- to late-1970s saw the first major automation advances over paper peak measurement calculations and they came from two directions:

- Expensive mini-computers allowing a central data system approach to multiple-channel data collection and storage;

- Cheaper single-channel computing integrators with little or no data storage capability initially, followed by increased capability to capture data from two or four channels (chromatographs);

- This evolutionary bifurcation or split in the development of CDS was to continue during the 1980s, with the emergence of the IBMbased PC-based CDS, which we will discuss in the Iron Age section of this paper.

\section{- Central data systems}

Centralized data systems based on a host computer and data-acquisition channels were developed commercially by the mid-1970s for larger laboratories. This was typified by the Hewlett Packard 3350 series data system with an HP 1000 host computer linked to multiplexed analogue to digital devices for data collection and, later in the 1980s, a host computer being a Digital Equipment Corporation VAX. Although relatively expensive, these central data systems allowed a laboratory to store and share both methods and data files. Graphical reprocessing was possible, which had the advantage of reducing the need for some sample re-injection.

Using a programming language, the data system functions could be expanded to include calculation of calibration curves and the calculation of the analyte concentration in unknown samples. However, the user was responsible for these additions and sometimes a CDS vendor would host a library of user-developed routines or programs for others to access and use. The problem with user-developed programs was that typically these were not developed or tested following a recognized life cycle nor under change control as computer validation had yet to be considered by the regulators.

Following the development of client/server architectures, this type of CDS had been largely superseded by or had evolved into scalable PC network systems by the Technology Age.

\section{- Computing integrators}

The late 1970s also saw the introduction of computing integrators. Initially, they were single channel instruments that could take the detector and calculate the peak areas, which were printed out on thick rolls of paper, although plotting a chromatogram was not possible. The CDS program was located in firmware or read-only memory chips. The signal from the detector was fed into the integrator and parameters for data acquisition and processing were entered by the user for the integrator to calculate. Injection of samples was either manual or automatic via an autosampler but, in either situation, a contact closure signal would tell the integrator when the injection has been made, in order to start the run synchronously with the injector. The raw data from early integrators consisted of a combination of the chart recorder output for the run plus the integrator output annotated by the bioanalyst.

Memory limitations with the early integrators meant that only the current chromatogram was stored and, if you wanted to replot it or change integration parameters, you had to do that before the next injection wiped the current injection out of the memory. Manufacturing issues meant 
that the early integrators had a tendency to malfunction during an injection and it was not unknown for a bioanalyst to have to remove the back of the integrator and remove the printed circuit board in order to press all the chips into place to ensure the integrator returned to operational use. Later, more reliable, integrators could provide data acquisition from up to four chromatographs and save methods, but not data, on minitape cassettes. The major disadvantage of these multiple channel integrators is that injections from an individual instrument were not separate but were intermingled with output from other instrument data; so a pair of scissors was necessary to separate the integrated results from a single instrument run. This was not the most convenient arrangement and many laboratories used single-channel integrators in preference.

Driven by pharmaceutical manufacturing quality control laboratory needs, later integrators provided the capability to plot the chromatogram, thus eliminating the need for a chart recorder and allowing the chromatogram and integration to be placed next to one another. However, the quality control laboratory calibration requirements were not those of the bioanalyst and user-specified programs for calculation of regression lines were often required or calculation was performed in a separate computer system. The most sophisticated integrators at the end of the Bronze Age could also be networked together to share user-written programs and data, however data storage was limited, due to expensive solid-state memory or hard drive space.

\section{- Summary of Bronze Age CDS}

Typically, the CDS systems used in the Bronze Age were able to carry out data collection and peak measurement but there was no instrument control, which had to be carried out by setting up the chromatograph modules manually. Linkage between the autosampler and the CDS (integrator or central system) was via a contact closure upon injection of a sample as a trigger for the data system to start work on that injection. Often standard curve and unknown calculations had to be performed outside of the integrators as bioanalytical calibration was different from that used in manufacturing quality control laboratories and this was performed either by a separate computer program or a calculator. However, the computing integrator branch of CDS evolution was effectively made extinct by the coming of the personal computer.

\section{Iron Age: expansion to include instrument control}

The arrival of the PC in the early 1980s led to the introduction of PC-based CDS within 1-2 years. In addition, by the middle of the decade, instrument vendors had developed CDS systems running on the $\mathrm{PC}$ that could control their own hardware and provide an integrated solution to the customer. The PC brought the ability for the laboratory to become independent from corporate IT and many semi-autonomous laboratory networks were established at this time. In addition, as there were few IT standards for computers used in the laboratory, some vendors developed or users purchased Apple computers if there was CDS software that could run on one.

\section{Standalone PCs: extension to instrument control}

The operation of the early CDS systems running on a single standalone $\mathrm{PC}$ was similar to the integrator but data files from all injections of a run were now available for reprocessing on the hard drive with the PC that acquired the data. To reduce the cost per channel, PCs could provide instrument control as well as acquire data for typically two, but also up to four, chromatographs. Whilst an attractive option for some laboratory managers, keyboard contention first thing in the morning could be a problem and in many laboratories, control of more than one chromatograph was not a practical option for bioanalysis. Although there were the electronic data files available on the hard drive, paper was considered the raw data and often the electronic files produced were not backed up effectively and were often lost or even deleted to save still relatively expensive disk space.

\section{- PC client-server networks}

Advances in computing, such as easy availability of relatively cheap and high-performing PCs and networking, led to the availability of networked client-server networks. These and the single PC workstations tend to be the approach used today. As a result of these developments, the central computer CDS based on a mini-computer started on the path to extinction.

The great advantage of the client-server networks was the ability to store and share data centrally on the corporate network. Furthermore, backup of the system was performed regularly by the IT professionals and taken out of the hands of laboratory staff who had a tendency to perform ad-hoc backups. Although this was 
a trend in mainstream CDS for quality control, it developed later in CDS for bioanalysis, and it was not until the Technology Age that it came for LC-MS systems.

\section{Summary of Iron Age CDS}

This is the Age of CDS evolution where the modern CDS begins to be seen. In the earliest forms, the data system could run on a variety of personal computer hardware platforms; however, by the end of the Iron Age, it was apparent that the PC has become the dominant technology and corporate IT began to reassert corporate standards, even in the laboratory. The problem is that, although the system works effectively, changes in regulations impacting bioanalysis appeared at the end of the Iron Age, impacting the regulatory compliance features of the CDS systems: 21 CFR 11 on electronic records and signatures [4].

\section{Technology Age: electronic working \& regulatory compliance}

Evolution of CDS in the Technology Age is less about the technology platform used to deliver the application and more about the functions built into the system for electronic working and compliance with current regulations. The technology platform choice for the bioanalyst is either several standalone systems or a single networked system. The networked system either just stores data to a single networked file server (to allow retrieval to the instrument workstation where the data were originally acquired) or the introduction of processing workstations within the network (to free up the instrument for more data acquisition), thus reducing keyboard contention and increasing throughput.

\section{- Migrating from paper to electronic records}

Until the issue of the final version of 21 CFR 11 in 1997 [4], the raw data definition in all bioanalytical laboratories was essentially the paper printouts of processed chromatograms with calculated results. With the introduction of Part 11, the transition from paper raw data to electronic record raw data began, but not every laboratory was happy to make this migration. However, there is no doubt that raw data in the case of CDS are electronic records and much time was wasted by many laboratories trying to justify that this was not the case. AGIT, the Swiss working group on IT, have written two very good guidance documents on definition of electronic records, the archiving of them [101] and the Organisation for Economic Cooperation and Development definition of electronic archives [5], which should be read by all concerned about electronic records.

\section{- Part II regulatory compliance features}

As an additional result of 21 CRF 11 regulations, all CDS sold by companies to the pharmaceutical industry needed to implement the technical controls to ensure compliance with the final rule. Some of these additional features included:

- Further development of the existing access controls (authority checks) to allow greater granularity, especially around access to electronic signatures and configuration of the system by authorized individuals;

- Removal of the audit trail from within the chromatography data file where it was unable to detect its own deletion and hence fail to comply with Part 11. Expansion of audit-trail functionality to cover all aspects of operator activities within the system and include the ability of users to enter either free text or predefined comments about the reason for change to comply with the underlying GLP predicate rule;

- Protection of all electronic records (e.g., the meta data, such as processing method and sequence file) as well as the raw data file to prevent tampering with the record and the automatic linkage with the audit trail when changes to files are detected by the system;

" Implementation of electronic signatures.

However, as the Part 11 world was continually evolving, CDS vendors went through a continuous cycle of interpretation, along with the pharmaceutical industry, and it typically took two or more versions of a single application before a stable interpretation of 21 CFR 11 technical functions was implemented in the majority of CDS applications used in bioanalysis.

\section{- Compliant electronic working practices}

As a consequence of implementing Part 11 technical controls in CDS application, arose the ability to reduce, if not eliminate, the generation of paper in the bioanalytical laboratory. However, as most of the industry, including quality assurance units, is ultra conservative the 
speed of implementing this could be very slow although there are substantial business benefits to be obtained from this transformation [2].

\section{Summary of Technology Age CDS}

The development of Part 11 technical controls has been a salutary learning experience for both CDS vendors and the bioanalysts who had to implement compliant working practices. Development of the technical controls required was an iterative process and had a fair amount of misunderstanding and misinterpretation as both vendors and laboratories had many different approaches, driven mainly by GMP rather than GLP. However, there is now a good consensus for interpretation of the current regulation, although as Part 11 is due to be updated soon, this picture may change.

\section{Summary}

We have seen, over the past 40 years and four ages of CDS evolution, a move from a completely manual process to a semi-automated or sometimes electronic process, where the definition of raw data has changed from paper to electronic media. This has been an interesting journey that has sometimes been relatively straightforward and sometimes painful; however, the current CDS systems are infinitely better than working manually.

- Some words of warning...

Chromatography data systems are very useful in automating the quantification of analytes and are readily accepted as de facto requirements in all bioanalytical laboratories that use chromatographic separations in one form or another. However,

- Are things as good as they appear?

- How much do we know about the software functions that we use on a daily basis?

- How much do we take on trust just because a computer says that these are the results?

- Do we really understand what a CDS does and the results it produces?

Data generated from any CDS system must be understood and the limitations of each different application known. There is an implicit belief that data generated by a CDS are trustworthy and reliable, especially if they are backed up with numbers expressed to six or more decimal places - nothing can be further from the truth! Never believe that a CDS can save you from separating the analyte from partially unresolved peaks. Extensive reintegration by a CDS must never be a substitute for poor chromatographic separation. Baselines and peak-measurement algorithms use the same principles as manual quantification techniques. The only difference is that with a CDS these are automated and you believe they work!

\section{Quo vadis CDS?}

Where are we going in the future with CDS? In my view, there is only one way and this is fully electronic, where the CDS applications and systems used by bioanalysts are integrated with LIMS, and current paper laboratory notebooks are replaced with an electronic medium. However, the current CDS systems are really not fit for complete electronic working and this will usher in the fifth age of CDS: the Electronic Age.

We need electronic workflows to be defined in CDS so that when an analytical batch or the complete set of results for a study are ready for review, the supervisor knows this electronically, either when they log on to the CDS or via an e-mail sent by the application. The workflow will define the bioanalytical staff who will be working on each study and the equipment used. Data will be input from LIMS but also electronic laboratory notebooks. When each member of staff logs on, there will be a list of the work they have to perform for each study. Supervisors will have configurable and visible flags available to help review each analytical run, for example to indicate how many times an injection has been reintegrated or whether the baselines have been changed. At the moment, this information is available but a user has to hunt for it within the CDS, this is not the way it should be - the system should just present this for the supervisor to check. Similarly, analysis of the standard curve and quality control samples can be similarly presented ready for review by the bioanalyst and their supervisor.

Financial \& competing interests disclosure The author has no relevant affliations or financial involvement with any organization or entity with a financial interest in or financial conflict with the subject matter or materials discussed in the manuscript. This includes employment, consultancies, honoraria, stock ownership or options, expert testimony, grants or patents received or pending, or royalties.

No writing assistance was utilized in the production of this manuscript. 


\section{Bibliography}

1 Dyson N. Chromatographic Integration Methods, 2nd Edition. Royal Society of Chemistry, Cambridge, UK (1999).

2 McDowall RD. Validation of Chromatographic Data Systems, Meeting Business and Regulatory Requirements. Royal Society of Chemistry, Cambridge, UK (2005).

3 Shah VP, Midha KK, Dighe S et al. Bioanalytical Method Validation Conference Report. Pharm. Res. 9, 588-592 (1992).
4 Department of Health and Human Services. Electronic Records; Electronic Signatures, final rule. Federal Register. Title 21 CFR Part 11 of the Code of Federal Regulations. 20 March 1997.

5 Organisation for Economic Cooperation and Development (OECD). Establishment and control of archives that operate in compliance with the principles of GLP. OECD Series $O n$ Principles Of Good Laboratory Practice And Compliance Monitoring Number 15. OECD (2007).

\section{- Website}

101 Guidelines for the Acquisition and Processing of Electronic Raw Data in a GLP

Environment, AGIT, 2005. Guidelines for the Archiving of Electronic Raw Data in a GLP Environment, AGIT 2003 www.bag.admin.ch/themen/ chemikalien/00253/00539/03300/index. html?lang=en 